\title{
Filling factor and temperature variations in planetary nebulae
}

\author{
Manuel Peimbert and Antonio Peimbert \\ Instituto de Astronomía, Universidad Nacional Autónoma de México, \\ Apartado postal 70-264, México D. F. 4510, México \\ email: peimbert@astroscu.unam.mx; antonio@astroscu.unam.mx;
}

\begin{abstract}
We have found a strong correlation between small filling factors and large $t^{2}$ values in planetary nebulae. We have also found that in general the filling factor for Type I PNe is smaller than for Type II PNe. These results imply that the abundance correction due to temperature inhomogeneities in general is larger for Type I PNe than for Type II PNe. This difference permits to reproduce the expected abundance difference between PNe of Type I and II predicted by Galactic chemical evolution models.
\end{abstract}

Keywords. ISM: abundances, planetary nebulae: general; Galaxy: abundances

\section{Discussion}

To test the abundance determinations of PNe we will compare the Galactic chemical evolution model by Carigi \& Peimbert (2011) with the abundances derived from collisionally excited lines for PNe of Type I and Type II. To make this comparison we have to address several problems. We need to estimate: a) the correction of the $\mathrm{O} / \mathrm{H}$ value due to the temperature structure of the nebulae, b) the fraction of $\mathrm{H}$ that is converted into He during the central star evolution, c) the fraction of $\mathrm{O}$ trapped by dust grains in the nebulae and $d$ ) the average ages of the two types of PNe.

We present a preliminary discussion of the relationships for a set of PNe between: a) the abundance discrepancy factor, ADF, versus the radius of the nebulae, b) the filling factor, defined by $\epsilon=N_{e}(\mathrm{rms})^{2} / \mathrm{N}_{\mathrm{e}}(\mathrm{FL})^{2}$, versus the ADF values, and c) $\epsilon$ versus radius.

We are in the process of increasing the number of data points with accurate $\epsilon, t^{2}$ (Peimbert 1967), and abundance values. The sources of error, that in some cases are not independent, include the following: the angular radius $\theta$ (e. g. Mallik \& Peimbert 1988), the distance to the object, the average root mean square density $\left\langle N_{e}(\mathrm{rms})\right\rangle$, the average forbidden line density $\left\langle N_{e}(\mathrm{FL})\right\rangle$, the reddening correction $C(\mathrm{H} \beta)$, the $\mathrm{ADF}$ that includes the errors in the line intensities, and the average $t^{2}$ value.

In Table 1 we compare the Galactic chemical evolution model by Carigi \& Peimbert (2011) with: a) the HII region abundances of the solar vicinity derived form RLs by Esteban and collaborators corrected by the amount of $\mathrm{O}$ embedded in dust grains according to Peimbert \& Peimbert (2010), b) the metal richest F and G stars of the solar vicinity studied by Bensby \& Feltzing (2006), c) the protosolar abundances presented by Asplund et al. (2009), and d) the abundances for PNe of Types I and II presented in the literature and in this poster.

\section{Conclusions}

1) We find a strong correlation between the ADF value and the filling factor, or $t^{2}$ and $\epsilon$. The smaller values of $\epsilon$ imply larger density variations, and in the presence of larger density variations we expect larger temperature variations. 
Table $1.12+\log \mathrm{O} / \mathrm{H}$ values.

\begin{tabular}{ccccccc}
\hline $\begin{array}{c}\text { Age } \\
\text { (Gyr) }\end{array}$ & G.C.E. $^{a}$ & $\begin{array}{c}\text { Type I PNe } \\
\text { (CELs) }\end{array}$ & $\begin{array}{c}\text { Type I PNe } \\
\text { (RLs) }\end{array}$ & $\begin{array}{c}\text { Type II PNe } \\
\text { (CELs) }\end{array}$ & $\begin{array}{c}\text { Type II PNe } \\
\text { (RLs) }\end{array}$ & Other \\
\hline 0 & 8.88 & $\ldots$ & $\ldots$ & $\ldots$ & $\ldots$ & $8.87^{b}$ \\
1 & 8.84 & $8.57^{c}$ & $9.14^{d}$ & $\ldots$ & $\ldots$ & $8.84^{e}$ \\
4.5 & 8.68 & $\ldots$ & $\ldots$ & $\ldots$ & $\ldots$ & $8.73^{f}$ \\
6 & 8.58 & $\ldots$ & $\ldots$ & $8.58^{c}$ & $8.87^{d}$ & $\ldots$ \\
\hline
\end{tabular}

${ }^{a}$ Galactic chemical evolution model, solar vicinity, Carigi \& Peimbert (2011).

${ }^{b}$ Solar vicinity H II regions, Carigi \& Peimbert (2011).

${ }^{c}$ Solar vicinity PNe, Stanghellini \& Haywood (2010) and Henry et al. (2010).

${ }^{d}$ This work.

${ }^{e}$ Young F and G stars of the solar vicinity, Bensby \& Feltzing (2006).

${ }^{f}$ Protosolar value, Asplund et al. (2009).

2) From Galactic chemical evolution models it is found that $\langle z\rangle$ is about $100 \mathrm{pc}$ for Type I PNe (about one Gyr old) and about 280 pc for Type II PNe (3-9 Gyr old), see Allen et al. (1998) and García-Segura et al. (2002). Furthermore it is also found that the $\mathrm{O} / \mathrm{H}$ value of the ISM when Type I PNe were formed was about 0.25 dex higher than when Type II PNe were formed.

3) We find that the ADF for Type I PNe is about 0.25 dex higher than for Type II $\mathrm{PNe}$. In other words that from recombination lines it is found that the $\mathrm{O} / \mathrm{H}$ value for Type I PNe is about 0.25 dex higher than for Type II PNe.

4) Stanghellini \& Haywood (2010) and Henry et al. (2010) find that for the solar neighborhood the $12+\log \mathrm{O} / \mathrm{H}$ values for Type I and Type II PNe derived from CELs are practically the same and amount to about 8.58. Rodríguez \& Delgado-Inglada (2011) find that for 8 well observed PNe of Type II of the solar vicinity the $12+\log \mathrm{O} / \mathrm{H}$ average value for CELs amounts to 8.68 and for RLs amounts to 8.98.

5) We find that the $\mathrm{O} / \mathrm{H}$ values for Type I and Type II PNe derived from RLs are about 0.3 dex higher than those predicted by Galactic chemical evolution models. This discrepancy should be studied further.

\section{References}

Allen, C., Carigi, L., \& Peimbert, M. 1998, ApJ, 494, 247

Asplund, M., Grevesse, N., Sauval, A. J., \& Scott, P. 2009, ARAEAA, 47, 481

Bensby, T. \& Feltzing, S. 2006, MNRAS, 367, 1181

Carigi, L. \& Peimbert, M. 2011, RevMexAA, 47, 139

García-Segura, G., Franco, J., López, J. A., Langer, N., \& Rózyczka, M. 2002, RevMexAASC, 12,117

Henry, R. B. C., Kwitter, K. B., Jaskot, A. E., Balick, B., Morrison, M. A., \& Milingo, J. B. 2010, ApJ, 724, 748

Mallik, D. C. V. \& Peimbert, M. 1988, RevMexAA, 16, 111

Peimbert, A. \& Peimbert, M. 2010, ApJ, 724, 791

Peimbert, M. 1967, ApJ, 150, 825

Rodríguez, M. \& Delgado-Inglada, G. 2011, ApJ, 733, L50

Stanghellini, L. \& Haywood, M. 2010, ApJ, 714, 1096 